\title{
AC: Electrostatic lubrication of natural joints
}

\author{
Raghuvir Pai* \\ Department of Mechanical and Manufacturing Engineering, Manipal University, Manipal, India
}

\begin{abstract}
This monograph attempts to explain a new joint lubrication mechanism with surface active phospholipids as a lubricant. It provides studies of the principles of cartilage (smart material) of biological micro- and nanotribology.
\end{abstract}

The monograph consists of 160 text pages, 9 chapters and contains 95 figures' with about 300 bibliographic items in alphabetical order. The monograph is based on the author's own research and is very interesting because the subject of research is the surface of the natural joints. The author has determined the biophysical and biochemical parameters of articular cartilage and suggests a new mechanism of lubrication in natural joints. The Lamellar-repulsive joint lubrication mechanism is not so well-known in tribology and therefore deserves to be published.

The chemical and physical nature of the biological surfaces is seen in an entirely different light than that of engineering surfaces immersed in water. This book attempts to explain a new joint lubrication mechanism with surface- active phospholipids (PLs) as a lubricant. Joint lubrication is a complex problem and attributing macromolecules to the synovial fluid of the lubricant cannot fulfill all the functions. The self-organization process of a stable pore structure in phospholipid liposomes, bilayers, lamellar phases in the synovial fluid forces the lamellar-repulsive mechanism of lubrication. The lubricant is chemically attached to the surface and is responsible for the biological lubrication mechanism.

In an animal's body, where the tissues slide over each other, the lubrication mechanism has been referred to as a "lamellar-repulsive", because the surfaces coated with PL bilayers and lamellar phases negatively charged on articular surface with synovial fluid support this mechanism.

By giving an overview of physicochemical properties, the cartilage surface parameters like wettability and surface energy were determined using atomic force microscopy (AFM) and confocal microscopy (CFM). Friction of amphoteric and negatively charged cartilage surfaces $\left(-\mathrm{PO}_{4}^{-}\right)$ is also determined. Professor Pawlak has provided a comprehensive text to the biomechanics of frictional surfaces. The study provides the basis for the principles of biological micro- and nanotribology.

The amphoteric PLs are the main solid-phase components on the surface of an Articular Cartilage (AC). The liposomes were used as the model membrane to characterize the interfacial energy over the $\mathrm{pH}$ range 1 to 9.5, the isoelectric point (at $\mathrm{pH} \sim 4.20$ ) and amphoteric character of phospholipid bilayers. Cartilage phospholipidic membrane has been shown to undergo conformational reorientation when wettability changed from the wet $\left(\sim 0^{\circ}\right)$ super hydrophilic to dry-air $\left(103^{\circ}\right)$ hydrophobic state (named smart material).
It has been well established that low friction is supported by the PLs bilayers mechanism which essentially consists of a Surface Amorphous Layer (SAL) surrounded by a $0.155 \mathrm{M}$ electrolyte Synovial Fluid (SF) of $\mathrm{pH} \sim 7.4$ with high-molecular-weight charged biomacromolecules. The cartilage implication from osteoarthritis disease by a gradual erosion of the surface amorphous layer has shown an increased friction coefficient. The author in this book demonstrates experimentally that the cartilage smart biomaterial $\mathrm{pH}$ is sensitive to friction and introduces a novel concept in joint lubrication on charged surfaces.

The book is an excellent starting point for medical and biomechanical students and researchers working with biological systems. Also, it will be useful in areas of high technology and nano-material sciences. The reviewer is of the opinion that the book presents the original experimental results which very important for the understanding of joint lubrication and deserves all the respect for reading [1] (Figure 1).

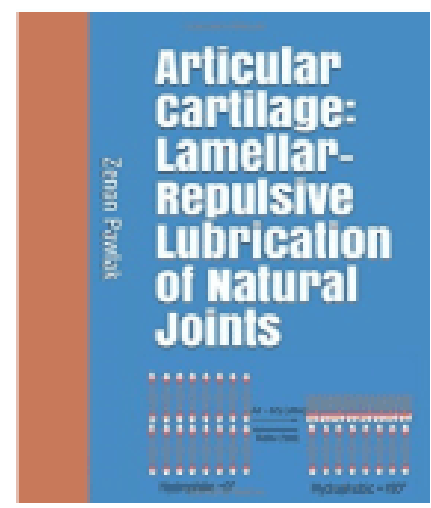

Figure 1. Cover page of the book

${ }^{\star}$ Correspondence to: Raghuvir Pai, Department of Mechanical and Manufacturing Engineering, Manipal University, Manipal, 576 104, India, E-mail: raghuvir.pai@manipal.edu

Key words: articular cartilage, natural lubrication, lamellar-repulsive mechanism of lubrication of natural joints

Received: December 19, 2018; Accepted: December 29, 2018; Published: December 31, 2018 


\section{CONTENTS}

1.0 Introduction

2.0 The structure, composition and function of cartilage 5

2.1 Synovial fluid

2.2 Phospholipid bilayers as a potential solid lubricant 17

2.3 Phospholipids

2.4 Cartilage and antiphospholipid antibody syndrome 25

3.0 Surface cartilage characteristics

3.1 Interfacial energy of PL bilayers

3.2 Cartilage surface wettability vs. $\mathrm{pH}$

45

3.3 Friction vs. wettability of cartilage surface

3.4 Amphoteric cartilage and its consequences
3.5 Cartilage surface charge density vs. $\mathrm{pH}$

4.0 Porosity of lubricated cartilage surface 85

5.0 The boundary and boundary-layered lubrication

89 6.0 Lamellar-repulsive mechanism of low friction in Nature 93

7.0 Resurfacing cartilage surface 107

8.0 Articular cartilage restoration and regeneration $\quad 123$

9.0 Summary and outlook $\quad 131$

About the Author 137

Bibliography 139

Index 153

\section{References}

1. Pawlak, Zenon (2018) Articular Cartilage: Lamellar-Repulsive Lubrication of Natural Joints, Kindle Direct Publishing, 171pp. Print-book: https://www.amazon.com/dp/ B07B42P1JY, e- book: https://www.amazon.com/dp/1976760283.

Copyright: (2018 Pai R. This is an open-access article distributed under the terms of the Creative Commons Attribution License, which permits unrestricted use, distribution, and reproduction in any medium, provided the original author and source are credited. 